\title{
NIMA-related kinase 2 regulates hepatocellular carcinoma cell growth and proliferation
}

\author{
XIAO-BO LAI ${ }^{1,2}$, YU-QIANG NIE ${ }^{1,2}$, HONG-LI HUANG ${ }^{1,2}$, YING-FEI LI ${ }^{1,2}$, \\ CHUANG-YU CAO ${ }^{1,2}$, HUI YANG ${ }^{3}$, BO SHEN $^{1,2}$ and ZHI-QIANG FENG ${ }^{1,2}$ \\ ${ }^{1}$ Department of Gastroenterology and Hepatology, The First Municipal People's Hospital of Guangzhou, \\ Guangzhou Medical University; ${ }^{2}$ Guangzhou Digestive Disease Center, Guangzhou First People's Hospital, Guangzhou, \\ Guangdong 510180; ${ }^{3}$ Department of Gastroenterology, The Second Affiliated Hospital, \\ Guangzhou Medical University, Guangzhou, Guangdong 510282, P.R. China
}

Received January 27, 2016; Accepted October 27, 2016

DOI: $10.3892 / \mathrm{ol} .2017 .5618$

\begin{abstract}
NIMA-related kinase 2 (Nek2) is often upregulated in human cancer and is important in regulating the cell cycle and gene expression, and maintaining centrosomal structure and function. The present study aimed to investigate the expression pattern, clinical significance, and biological function of Nek2 in hepatocellular carcinoma (HCC). mRNA and protein levels of Nek2 were examined in HCC and corresponding normal liver tissues. The MTT and soft agar colony formation assays, and flow cytometry were employed to assess the roles of Nek2 in cell proliferation and growth. In addition, western blot analysis was performed to assess the expression of cell cycle- and proliferation-related proteins. The results revealed that Nek2 was upregulated in HCC tissues and cell lines. The clinical significance of Nek2 expression was also analyzed. Inhibiting Nek2 expression by siRNA suppressed cell proliferation, growth, and colony formation in hepatocellular carcinoma cell line HepG2 cells, induced cell cycle arrest in the G2/M phase by retarding the S-phase, and promoted apoptosis. Furthermore, Nek2 depletion downregulated $\beta$-catenin expression in HepG2 cells and diminished expression of Myc proto-oncogene protein (c-Myc), cyclins D1, B1, and $\mathrm{E}$ and cyclin-dependent kinase 1, whilst increasing protein levels of $\mathrm{p} 27$. This demonstrates that overexpression of Nek2 is associated with the malignant evolution of HCC. Targeting Nek2 may inhibit HCC cell growth and proliferation through the regulation of $\beta$-catenin by the $\mathrm{Wnt} / \beta$-catenin pathway and therefore may be developed as a novel therapeutic strategy to treat HCC.
\end{abstract}

Correspondence to: Dr Yu-Qiang Nie, Department of Gastroenterology and Hepatology, The First Municipal People's Hospital of Guangzhou, Guangzhou Medical University, 1 Panfu Road, Yuexiu, Guangzhou, Guangdong 510180, P.R. China E-mail: yuqnie@163.com

Key words: hepatocellular carcinoma, Nek2, cancer proliferation, wnt $/ \beta$-catenin

\section{Introduction}

Hepatocellular carcinoma (HCC) is the third most common cancer worldwide (1). Each year, $>620,000$ patients are diagnosed with HCC, and the 1-year survival rate remains $<50 \%$ (2). Over the past few decades, HCC age-adjusted incidence rates have doubled (3), and primary liver cancer mortality rates have increased faster than the mortality rates of most other cancers (4).

Current therapeutic modalities for HCC include curative options, such as surgical resection, liver transplantation, and local ablation; or palliative procedures, such as catheter-directed therapies and systemic therapy (5). The main risk factors for HCC are viral hepatitis, alcohol abuse, aflatoxin B1-contaminated food, nonalcoholic fatty liver disease, and metabolic disorders (6). However, not all individuals exposed to these factors possess the same risk of developing HCC. It is a multifactorial disease, with a wide range of genetic and epigenetic alterations identified as contributory to the deregulation of key oncogenes and tumor-suppressor genes; however, genetic events in hepatic carcinogenesis are poorly understood (7). Previous studies undertaken by our group uncovered potential molecular targets for HCC treatment, by comparing the gene expression patterns of HCC with those of normal liver tissues using a cDNA expression microarray containing 1361 unique genes (8). Among the genes explored, NIMA-related kinase 2 (Nek2), also known as Serine/threonine-protein kinase 2, was identified as a potential target gene as it is highly expressed in HCC.

Nek2 is a member of the serine/threonine kinase family, and is important in regulating the cell cycle, gene expression, and maintaining centrosomal structure and function (9-11). Nek2 belongs to the never in mitosis A (NIMA)-related family of kinases, otherwise known as Neks (9). The Nek family comprises 11 members, all of which have been identified in mammals (9). Given the role of Nek2 in regulating centrosomal function during the $\mathrm{S}$ - and $\mathrm{G} 2$-phases, Nek2 regulation is strictly controlled throughout the cell cycle, with a hinged expression in such phases (12). Functional research has implicated Nek2 in the regulation of centrosome separation and spindle formation $(9,13)$. Furthermore, high Nek2 expression levels have been reported in cell lines derived from breast, 
cervical, prostate, and colorectal cancers, as well as those from cholangiocarcinoma and lymphoma (14-17). A recent study involving a cohort of normal liver tissues, chronic liver disease induced by the hepatitis $\mathrm{C}$ virus, and HCC, identified Nek2 co-expression with several closely related genes (18).

The present study aims to investigate Nek2 expression and biological regulation in human HCC, analyze the association of Nek2 expression with the clinicopathological characteristics of HCC patients, and examine the Nek2-mediated control of cell growth and proliferation to explore the potential molecular mechanisms involved in the progress of HCC.

\section{Materials and methods}

Patients and tissue samples. Patients were recruited from the First Municipal People's Hospital of Guangzhou, and clinical information was acquired from hospital registries. Primary tumor specimens were obtained from 52 patients diagnosed with HCC, all of whom provided informed consent for participation in the study. All 52 patients underwent routine resection in the First Municipal People's Hospital of Guangzhou between March 2010 and November 2012. HCC was diagnosed according to the World Health Organization histological classification of tumors of the liver and intrahepatic bile ducts (2000). All surgical specimens were immediately snap-frozen in liquid nitrogen and stored at $-80^{\circ} \mathrm{C}$ until RNA and protein extraction. The samples were obtained from tumor tissues and corresponding adjacent healthy tissues $5-10 \mathrm{~cm}$ from each tumor $<30 \mathrm{~min}$ following surgery. Clinical histopathological data including histopathological diagnosis, Edmondson-Steiner stage, and tumor grade were obtained from patient medical records. The present study was approved by the Ethics Committee of First Municipal People's Hospital of Guangzhou, Guangdong, China.

Cell culture and transfection. The human hepatocellular carcinoma cell lines of HepG2, Hep3B, BEL-7402, SMMC-7721, QCY-7701, PLC/PRF/5, and the human hepatocyte cell line HL-7702, were obtained from the cell repository of Sun Yat-sen University (Guangzhou, Guangdong, China). All cells were cultured at $37^{\circ} \mathrm{C}$ in a humidified atmosphere with $5 \%$ $\mathrm{CO}_{2}$ in Dulbecco's modified eagle medium (Gibco; Thermo Fisher Scientific, Inc., Waltham, MA, USA) containing 10\% fetal calf serum (Invitrogen; Thermo Fisher Scientific Inc.) and $100 \mathrm{IU} / \mathrm{ml}$ penicillin-streptomycin solution (Corning Inc., Corning, NY, USA). The cells were grown on sterilized culture dishes (Corning Inc.), harvested, and passaged with $0.25 \%$ trypsin-EDTA (Corning, Inc.) upon reaching $80 \%$ confluence. X-treme GENE siRNA Transfection Reagent (Roche Diagnostics $\mathrm{GmbH}$, Mannheim, Germany) was used for siRNA transfection according to the manufacturer's instructions. Protein levels were assessed $72 \mathrm{~h}$ later by western blot analysis, and mRNA levels were quantified $48 \mathrm{~h}$ later by reverse transcription-quantitative polymerase chain reaction (RT-qPCR). The anti-Nek2 siRNA (Sc43960; Santa Cruz Biotechnology Inc., Dallas, TX, USA) and control siRNA (Sc37007; Santa Cruz Biotechnology, Inc.) were used to transfect HepG2 cells.

Immunohistochemistry (IHC). IHC experiments were conducted on $5 \mu \mathrm{m}$ formalin-fixed, paraffin-embedded sections of healthy tissue and tumor samples. The tissue sections were mounted on slides, treated with xylene, and rehydrated using alcohol-water mixtures before heat treatment in $10 \mathrm{mM}$ citric acid (pH 6.0) for antigen retrieval. Endogenous peroxidase activity was stopped by the use of $0.3 \% \mathrm{H}_{2} \mathrm{O}_{2}$ solution. After blocking with $10 \%$ bovine serum albumin (Beyotime Institute of Biotechnology, Haimen, China; ST023) the sections were incubated at $4^{\circ} \mathrm{C}$ overnight with primary human Nek2 antibodies (Abcam, Cambridge, UK; Ab55550; 1:400 dilution), rinsed, and incubated with biotinylated anti-mouse $\mathrm{IgG}$ (Dako North America Inc., Carpinteria, CA, USA; F0232; 1:100 dilution) for $30 \mathrm{~min}$ at room temperature. After rinsing, the samples were treated with streptavidin and biotinylated horseradish peroxidase (Dako North America Inc.). Counterstaining with hematoxylin was performed, and the sections were dehydrated in ethanol before mounting. The sections were analyzed using optical microscopy (Nikon Corp., Tokyo, Japan; magnification, $\mathrm{x} 100$ or $\mathrm{x} 400)$. Staining specificity was confirmed using positive (gastric mucosa) and negative (heart muscle tissue) controls. Low Nek2 expression was defined as $<10 \%$ and high expression as $>10 \%$.

$R T-q P C R$. Total RNA was extracted from HepG2 cells or tissues using TRIzol (Invitrogen; Thermo Fisher Scientific, Inc.) according to the manufacturer's protocol. Complementary DNA was synthesized from $1.5 \mu \mathrm{g}$ of total RNA at $42^{\circ} \mathrm{C}$ for 60 min using the SYBR-Green PCR master mix (Thermo Fisher Scientific, Inc.; 4368577) and RT-qPCR was performed using the Brilliant II Sybr Green QPCR Master Mix (Agilient Technologies, Inc., Santa Clara, CA, USA). GAPDH was used as the reference gene. The primer sequences were as follows: Forward, 5'-CCAGCCCTGTATTGAGTG-3' and reverse, 5'-ACTTCCGTTCCTTTAGCA-3' for Nek2; forward 5'-GAC CTGACCTGCCGTCTA-3', and reverse, 5'-AGGAGTGGG TGTCGCTGT-3' for GAPDH. The relative levels of gene expression were represented as $\Delta \mathrm{Cq}$, and the fold-change of gene expression was calculated using the $2^{-\Delta \Delta \mathrm{Cq}}$ method.

Western blotting. The cells and tissue samples were lysed with $20 \mathrm{mmol} / \mathrm{l}$ Tris- $\mathrm{HCl}(\mathrm{pH} 8.0), 137 \mathrm{mmol} / \mathrm{l} \mathrm{NaCl}, 10 \%$ glycerol, $1 \%$ Triton X-100, and $2 \mathrm{mmol} / \mathrm{l}$ EDTA containing protease and phosphatase inhibitors at $4^{\circ} \mathrm{C}$. Each lysate sample $(20 \mu \mathrm{g})$ was separated by sodium dodecyl sulfate-polyacrylamide gel electrophoresis (SDS-PAGE) and transferred to nitrocellulose membranes. The membranes were incubated overnight at $4^{\circ} \mathrm{C}$ with antibodies against Nek2 (Santa Cruz Biotechnology, Inc.; Sc55601; 1:1,000 dilution), $\beta$-catenin, C-Myc, CycD1, CycB1, CDK1, CycE, and P27 (Santa Cruz Biotechnology, Inc., 1:1,000 dilution). The membranes were developed using enhanced chemiluminescence. The Nek2 expression levels were normalized to that of GAPDH, and were detected using mouse anti-GAPDH antibody (Santa Cruz Biotechnology, Inc.; Sc365062; 1:2,000 dilution).

Cell growth and proliferation. The cultured HepG2 cells were pelleted by centrifugation at $900 \mathrm{xg}$ and re-suspended in phenol red-free medium supplemented with $10 \%$ charcoal-stripped fetal bovine serum (HyClone; GE Healthcare Life Sciences, Logan, UT, USA) and 2\% L-glutamine. The HepG2 cells were then plated in triplicate, $\left(1 \times 10^{3}\right.$ cells $\left./ \mathrm{ml}\right)$ in 96 -well micro 

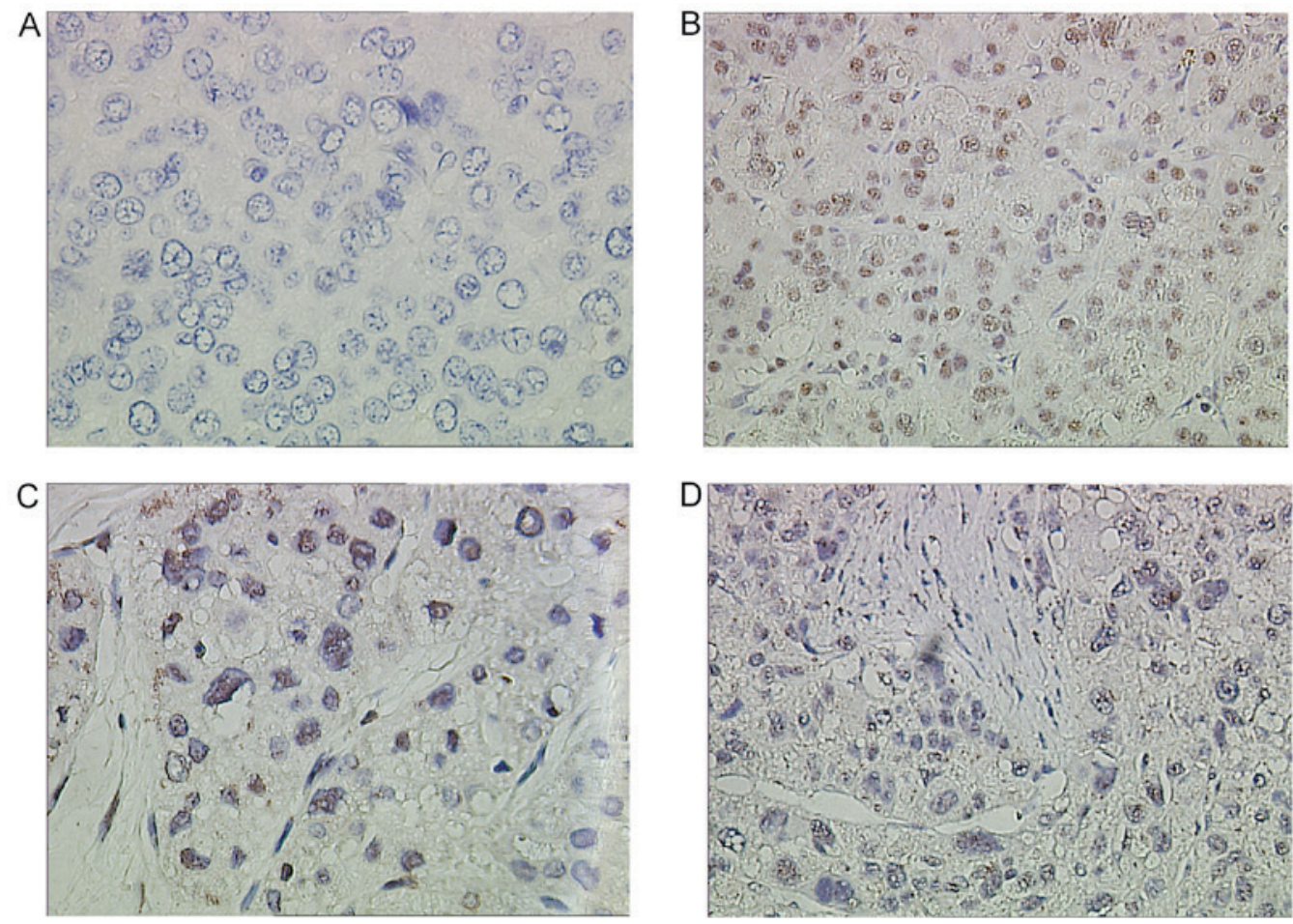

Figure 1. Immunohistochemical analysis of Nek2 in hepatocellular carcinoma (HCC) cells and adjacent non-cancerous tissue. (A) Negative Nek2 expression in HCC tissues. (B) Strong nuclear staining of Nek2 in a case of HCC Edmondson I. (C) Strong nuclear staining of Nek2 in a case of HCC Edmondson IIa. (D) Strong nuclear staining of Nek2 in a case of HCC Edmondson III. Negative nuclear staining was observed in all non-cancerous liver cells. In neoplastic cells, elevated levels of Nek2 were observed in the nuclei.

plates. This process was repeated six times. After seeding for $24 \mathrm{~h}$, the HepG2 cells were transfected with either anti-Nek2 or control siRNAs (10 $\mathrm{nM}$ each) using the X-treme GENE siRNA Transfection Reagent (Roche Diagnostics $\mathrm{GmbH}$ ) according to the manufacturer's protocol. The cells were counted daily over a 5-day period through the Cell Titer 96 Non-Radioactive cell proliferation assay (Promega Corp., Madison, WI, USA). Following this, $20 \mu \mathrm{l}$ of $5 \mathrm{mg} / \mathrm{ml}$ MTS solution was added to each well, and the resulting mixture was incubated at $37^{\circ} \mathrm{C}$ for $4 \mathrm{~h}$. Each solution was measured by a Bio-Rad micro plate reader (Bio-Rad Laboratories Inc.) at a test wavelength of $490 \mathrm{~nm}$.

Cell colony formation. In the anchorage-independent colony growth assay, the HepG2 cells were transfected with siRNA for $48 \mathrm{~h}$. A 12 -well cell culture plate containing $0.5 \%$ agarose medium on top of regular medium containing $1 \%$ low-melting agar was seeded with 500 transfected cells. After 15 days, the resulting colonies were stained with Giemsa and counted under a microscope.

Cell cycle and apoptosis analysis. HepG2 cells were seeded in $8 \mathrm{~cm}$ sterilized culture dishes for $12 \mathrm{~h}$. The cells were then transfected with indicated amounts of anti-Nek 2 or control siRNAs. At indicated time points, the cells were harvested, fixed with $1 \%$ paraformaldehyde, washed with phosphate buffer solution (PBS), and stained with $5 \mathrm{mg} / \mathrm{ml}$ propidium iodide (PI) in PBS supplemented with RNase A (Sigma-Aldrich, St. Loius, MO, USA) for $30 \mathrm{~min}$ at room temperature. Data were collected using a BD FACSCAlibur flow cytometer (BD Biosciences, San Jose, CA, USA). Apoptosis was measured using an
Annexin V/FITC kit (Sigma-Aldrich) according to the manufacturer's protocol. Each assay was repeated in triplicate.

Statistical analyses. All statistical calculations were performed using SPSS 13.0 software (SPSS Inc., Chicago, IL, USA). A chi-square test was used to examine possible correlations between Nek2 expression and clinicopathological factors. The differences in the densitometry data on focus numbers between the control and Nek2-transfected cells were analyzed using Student's t-test. $\mathrm{P}<0.05$ was considered to indicate a statistically significant result in both Student's t-test and the chi-square test.

\section{Results}

Nek2 expression in human HCC tissue and cell lines. Nek2 expression in resected human tissue specimens from healthy and cancerous livers was analyzed. Fifty-two HCC and normal samples were examined using Nek2-specific IHC staining antibodies (Fig. 1). The IHC results revealed clear differences in Nek2 expression between healthy and cancerous liver tissues. In the healthy and adjacent liver tissues, Nek2 was not expressed (Fig. 1A). By contrast, most HCC cases presented elevated protein levels in the nuclei (Fig. 1B-D). Expression of Nek 2 was examined in 17 pairs of snap-frozen HCC and adjacent nonmalignant liver tissues by western blotting. Levels of Nek2 protein were significantly elevated in the HCC specimens (T) compared with those in adjacent nonmalignant tissues (N; Fig. 2A). Meanwhile, analysis of Nek2 transcription by RT-qPCR and western blot analysis demonstrated that HepG2 cells expressed significantly higher levels of 
Table I. Association of Nek2 expression with clinicopathological characteristics of patients with HCC.

\begin{tabular}{|c|c|c|c|c|}
\hline Characteristics & Patients, no. & Nek2 positive, no. (\%) & $\chi^{2}$ & P-value \\
\hline \multicolumn{5}{|l|}{ Age, years } \\
\hline$<60$ & 17 & $11(64.7)$ & 0.632 & 0.426 \\
\hline$\geq 60$ & 35 & $19(54.3)$ & & \\
\hline \multicolumn{5}{|l|}{ Tumor size, $\mathrm{cm}$} \\
\hline$<5$ & 28 & $17(60.9)$ & 1.213 & 0.271 \\
\hline$\geq 5$ & 24 & $13(54.2)$ & & \\
\hline \multicolumn{5}{|l|}{ Hepatic function } \\
\hline Child A & 15 & $9(60)$ & 0.001 & 0.971 \\
\hline Child B & 37 & $21(56.7)$ & & \\
\hline \multicolumn{5}{|l|}{ Edmondson stage } \\
\hline Stage I and II & 42 & $22(52.4)$ & 4.434 & $0.037^{\mathrm{a}}$ \\
\hline Stage III and IV & 10 & $8(80)$ & & \\
\hline \multicolumn{5}{|c|}{ Portal vein tumor thrombus } \\
\hline Positive & 10 & $9(90)$ & 5.295 & $0.032^{\mathrm{a}}$ \\
\hline Negative & 42 & $21(50)$ & & \\
\hline \multicolumn{5}{|l|}{ AFP } \\
\hline Positive & 44 & $24(54.5)$ & 1.160 & 0.281 \\
\hline Negative & 8 & $6(75.0)$ & & \\
\hline
\end{tabular}

${ }^{\mathrm{a}} \mathrm{P}<0.05$. HCC, hepatocellular carcinoma; AFP, $\alpha$-fetoprotein.
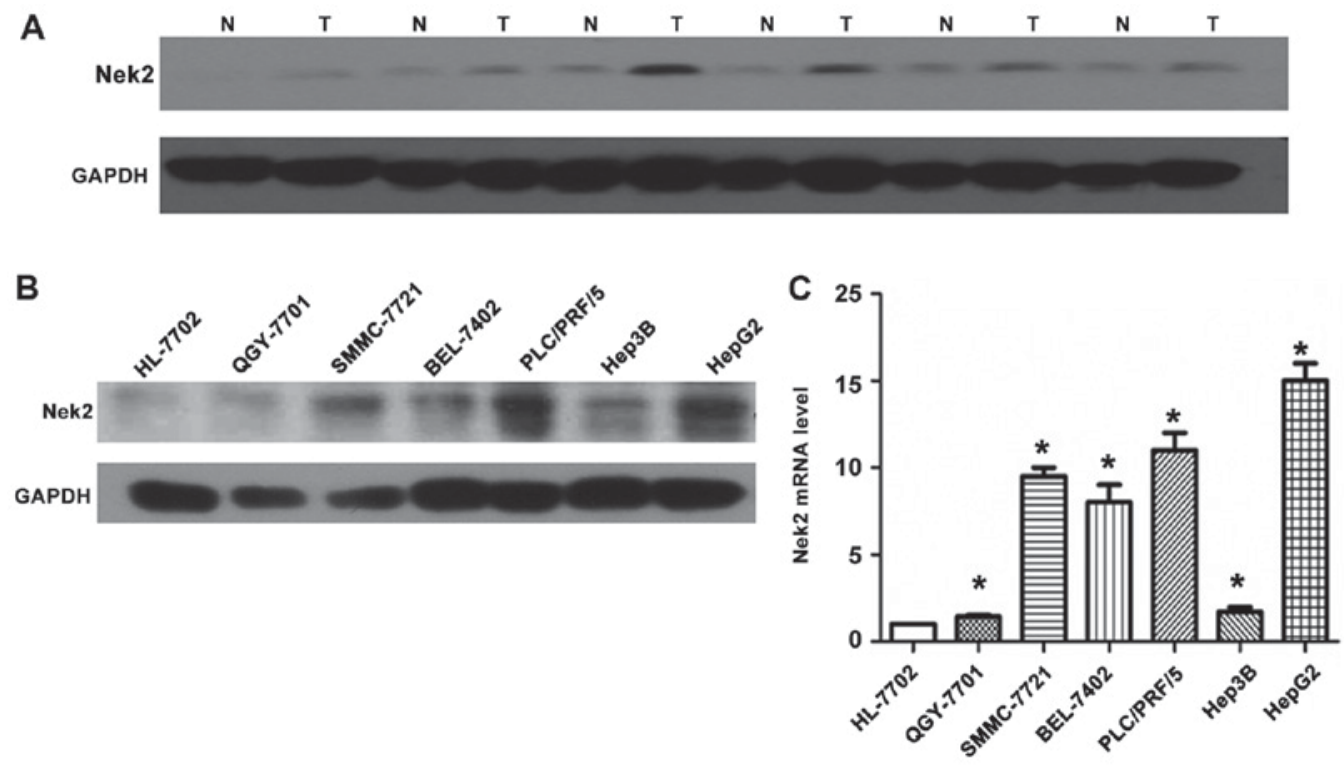

Figure 2. Expression levels of Nek2 in hepatocellular carcinoma (HCC), adjacent non-cancerous tissue, and HCC cell lines. (A) Expression levels of Nek2 protein in HCC tissues (T) were higher than in adjacent non-cancerous tissues (N). (B) Protein expression levels of Nek2 in a variety of HCC cell lines. (C) Nek2 mRNA levels in HCC cell lines. Both protein and mRNA levels of Nek2 were significantly higher in HCC than those in control (HL-7702 cell line; ${ }^{*} \mathrm{P}<0.05$, compared with HL-7702 cell line).

Nek2 mRNA and protein compared to the other six cell lines (HL-7702, PLC/PRF/5, Hep3B, BEL-7402, SMMC-7721, and QCY-7701; Fig. 2B and C).

Associations between Nek2 expression and the clinicopathological parameters of HCC patients. Thirty (57.6\%) out of 52 tumor tissues expressed Nek2 (Table I), and high levels of Nek2 were identified predominantly in the nuclei of cancerous liver cells. The correlation between Nek2 protein expression and the histological staging of liver cancer was analysed (Table I). Rates of Nek 2 expression in tumors classed as Edmondson-Steiner stages I and II (Fig. 1B and C) were $52.38 \%$ (22/42, Table I) and those with Edmondson-Steiner stages III and IV (Fig. 1D) were $80 \%$ (8/10; Table I). The 

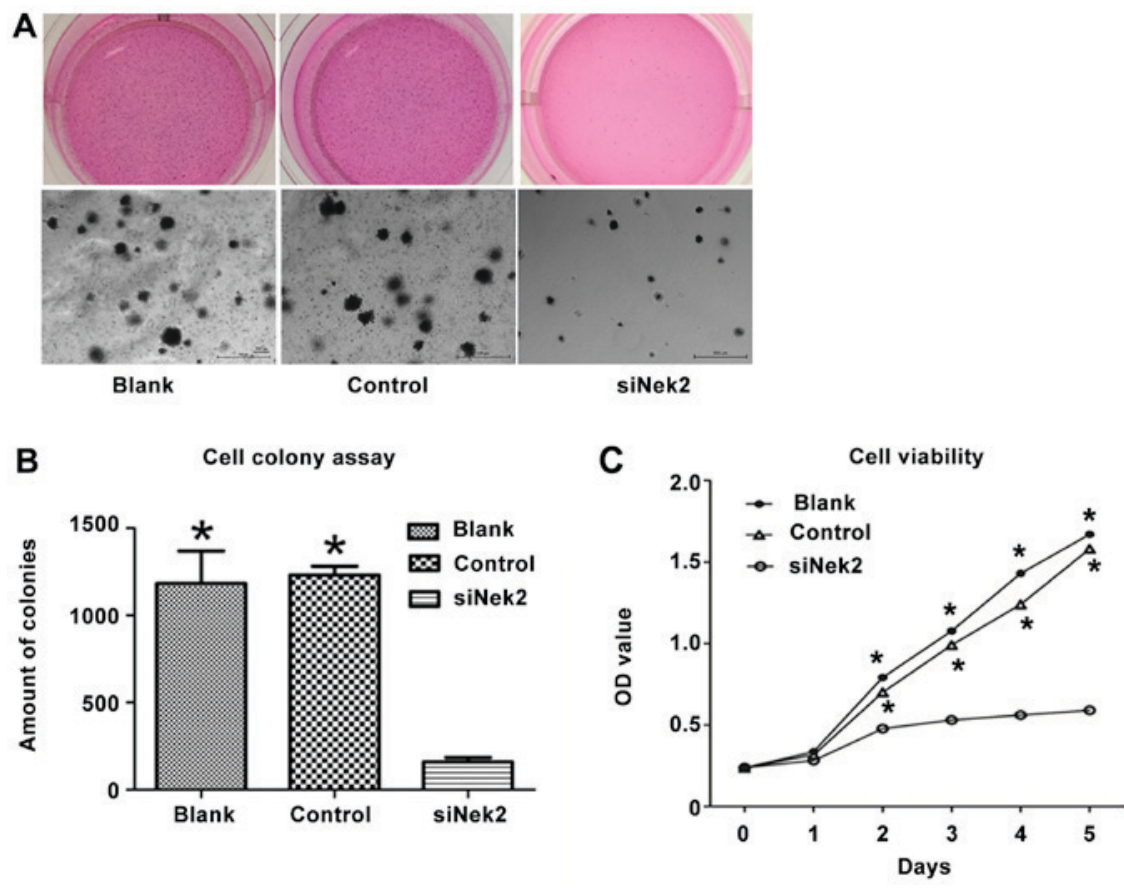

Figure 3. HepG2 cell proliferation and cell colony formation was down regulated by Nek2 inhibits. (A) Significant decreases in proliferation, (B) cell colony formation, and (C) cell viability were observed in HepG2 cells treated with Nek2 siRNA compared with cells transfected with control siRNA (control) and blank group (blank). ${ }^{\mathrm{P}}<0.05$, compared with siNek2 group.

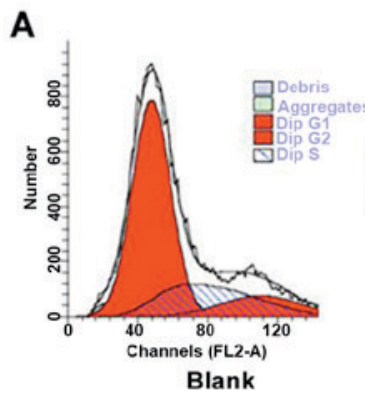

B

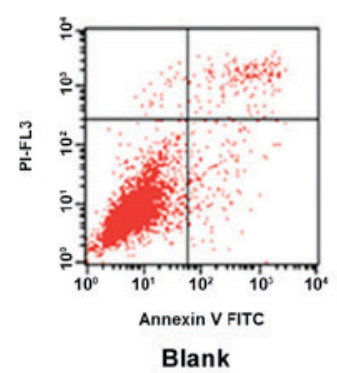

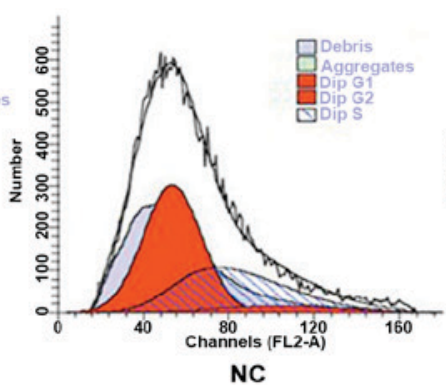

NC

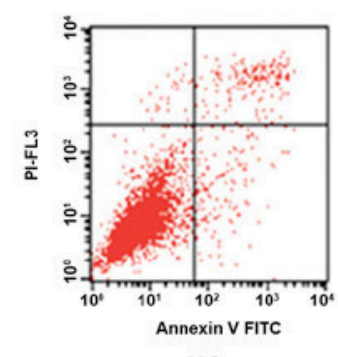

NC
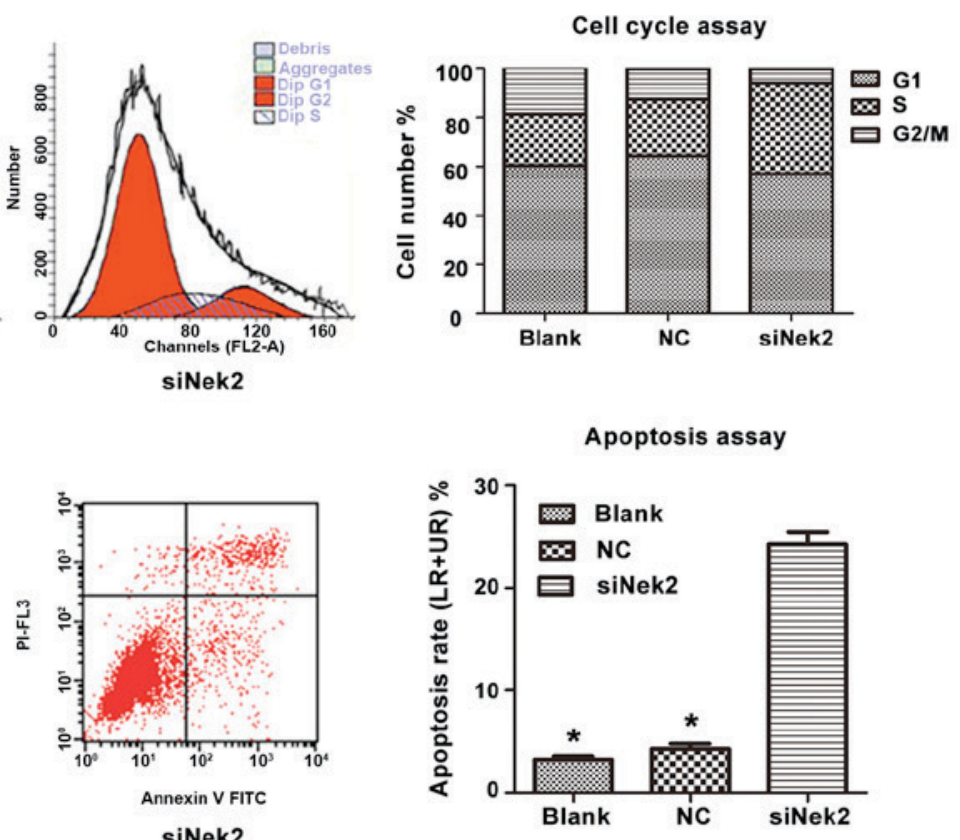

Figure 4. Effects of downregulating Nek2 on the cell cycle and apoptosis in HepG2 cells. (A) Nek2 knockdown increased the proportion of cells in the S-phase and decreased the proportion in the G2/M phase. Nek2 knockdown in HepG2 cells induces a cell cycle arrest in G2/M phase by slowing S-phase. (B) The apoptosis assay shows that HepG2 cells treated by Nek2 siRNA had a significantly higher apoptotic index than the control siRNA treated cells and blank cells. ${ }^{*} \mathrm{P}<0.05$, compared with siNek2 group.

difference in Nek2 expression between samples from the two tumor classes was statistically significant $(\mathrm{P}<0.05)$. Among all the samples, those with concomitant portal venous tumor emboli exhibiting Nek2 expression comprised 90.0\% (9/10), however, only $50.0 \%$ (21/42) without portal venous tumor emboli expressed Nek2 (Table I). A significant difference in Nek2 expression was noted in tumor malignancy $(\mathrm{P}<0.05)$.
Nek2 expression was observed to be independent of episode age, tumor size, Hepatic Function and liver $\alpha$-fetoprotein levels (Table I).

Inhibition of Nek2 suppresses HepG2 proliferation. Using siRNA as an efficient tool for Nek2 gene silencing in the HepG2 cell line, the role of Nek2 in HCC cell proliferation 


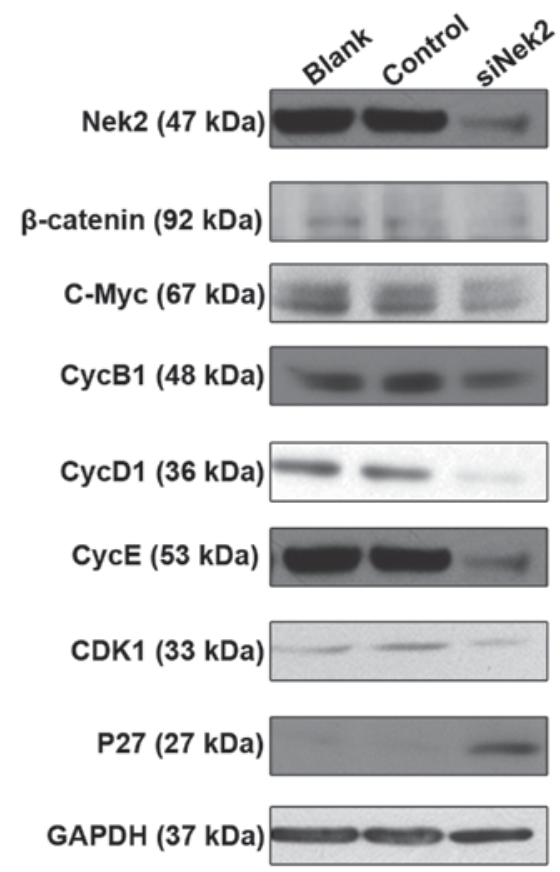

Figure 5. Western blot analysis of HepG2 cells. Western blotting revealed that Nek2 knockdown decreased protein levels of $\beta$-Catenin, c-Myc, CycD1, CycE, CycB1, CDK1, but increased p27 levels.

was analyzed. The proliferation rate was determined by MTS assay, assessing cell metabolic activity. A decrease in proliferation was observed in the HepG2 cells treated with anti-Nek2 siRNA compared with the cells transfected with the control siRNA and blank group (Fig. 3A). Consistent with the MTS result, the soft agar colony formation assays showed that Nek2 knockdown in HepG2 cells led to a significant decrease in focus number $[1183 \pm 97$ (Blank), $1232 \pm 28$ (Control) vs. $160 \pm 14$ (siNek2), P<0.05; Fig. 3B]. The cell viability of HepG2 cells treated with Nek siRNA decreased significantly compared with the control and blank groups ( $\mathrm{P}<0.05$; Fig. $3 \mathrm{C}$ ).

Effect of Nek2 on cell cycle and apoptosis of HepG2. The cell cycle of HepG2 cells transfected with either control siRNA or anti-Nek2 siRNA was analysed using PI staining followed by fluorescence-activated cell sorting. This revealed that the proportion of cells transfected with anti-Nek2 siRNA in the G2/M phase decreased. Furthermore, the proportion of cells treated with anti-Nek2 siRNA in the S-phase increased, whereas the proportion progressing through the G1/S-phase decreased (Fig. 4A). These observations suggest that Nek2 knockdown by specific siRNA in HepG2 cells induces cell cycle arrest in the G2/M phase by retarding the S-phase. Moreover, apoptosis analysis revealed that HepG2 cells treated with anti-Nek2 siRNA exhibited a significantly higher apoptotic index than controls (Fig. 4B).

Nek2 regulates $H C C$ growth through the $\beta$-catenin/Wnt pathway. The possible mechanisms of Nek2 involvement in $\mathrm{HCC}$ were detected by evaluating the protein levels of its established targets, including $\beta$-catenin, C-Myc, CycD1, CycB1, $\mathrm{CDK} 1, \mathrm{CycE}$, and P27 genes. These are all involved in the $\beta$-catenin/Wnt pathway and are essential for cell growth and proliferation. Western blot analysis indicated that $\beta$-catenin protein expression was compromised in HepG2 cells treated with Nek2-specific siRNA (Fig. 5). Furthermore, Nek2 inhibition resulted in the diminished expression of C-Myc, CycD1, CycB1, CDK1, and CycE proteins. Treatment of HepG2 cells with control siRNA did not influence the protein expression of any of the tested targets. However, western blot analysis also revealed that Nek2 knock down in the HepG2 cells led to increased P27 protein expression. Overall, these results suggest that Nek2 expression induces cell cycle progression and proliferation by regulating the $\beta$-catenin/Wnt pathway.

\section{Discussion}

The role of Nek2 in several human cancers has been explored previously. Nek2 expression is increased in breast, cervical, and prostate carcinoma cell lines, as well as in numerous cell lines established from lymphomas (19). An assessment of $\sim 1,700$ genes revealed marked up-regulation of Nek2 in cell lines derived from Ewing's tumor and apediatric osteosarcoma (20); and an increase in Nek2 mRNA levels was subsequently reported in non-Hodgkin lymphoma (21). Furthermore, in breast cancer models, Nek2 plays a role in tumorigenesis and drug resistance (22). However, though Nek2 is well established as an oncogene, its expression pattern and clinical significance remains poorly defined in human HCC.

The present study demonstrated that the cellular location of Nek2 was notably variable, with increased cytoplasmic expression with or without nuclear Nek2 accumulation. These differential results may be explained by Nek2's varying isoform expression, as its bipartite nuclear localization sequence spans the splice site (23). Isoform-specific antibodies may enable the expression and localization of specific Nek2 splice variants for further investigation in HCC tissues.

In the present study, Nek2 expression was increased in human HCC tissues, which has not been reported previously. HCC cells expressed elevated levels of Nek2, compared to the negligible levels detected in normal tissue cells. This may be due to the low Nek2 mRNA copy numbers in healthy tissue. High Nek2 expression was detected in 22 (52.4\%) Edmondson stages I and II cases, and in eight (80\%) of the Edmondson stages III and IV cases, suggesting that Nek2 is positively associated with more advanced HCC. Furthermore, Nek2 expression in low-stage HCCs was lower than that in high-stage HCCs. Meanwhile, other clinical association analyses demonstrated that expression of Nek2 was positively correlated not only with tumor histological grade and tumor metastasis, but also with hepatitis $\mathrm{C}$ viral infection, as has been reported previously (18). Thus, Nek2 may play an important role in HCC progression and serve as a potential biomarker for the biological behavior of HCC cells.

To elucidate the function of Nek2 in HCC cells, anti-Nek2 siRNA was transfected into the HepG2 cell line, which exhibited the highest Nek2 expression among all the seven HCC cell lines tested. Nek 2 knockdown decreased the proliferation rate and colony formation ability in HepG2 cells, suggesting that decreased Nek2 activity in HCC cells may decrease cancer cells' aggressive growth and increased proliferative properties. The latter hypothesis is supported by the established role of Nek2 in cell proliferation. In A549 lung adenocarcinoma cells and human embryonic kidney HEK293T cells, Nek2 
overexpression led to the activation of the Akt pathway and an increase in $\beta$-catenin protein levels (24). $\beta$-Catenin binds to and is phosphorylated by Nek2, and exists in a complex with Rootletin (25). In addition, the expression of CycD1, $\mathrm{CycE}$, and C-Myc genes, which are required for cell proliferation and regulated by $\mathrm{Wnt} / \beta$-catenin signaling, is controlled by Nek2 $(24,26)$. Consistent with these data, blocking Nek2 expression decreased levels of C-Myc, CycD1, and CycE1. The analysis of the underlying mechanisms of Nek2-mediated control of cell proliferation revealed that blocking Nek2 affected the cell cycle by retarding G2/M transition. This result was congruent with the expanded role of Nek2 as a regulator of $\mathrm{CycB} 1, \mathrm{CDK} 1$ and $\mathrm{P} 27$, all of which promote cell cycle progression and stimulate the G2/M transition.

Nek2 regulates the capacity for microtubule organization of the centrosome at the time of mitotic entry (26) and is involved in the regulation of chromatin condensation, mitotic checkpoint signaling, and cytokine activity (27). It may trigger centrosome separation at the onset of mitosis through the phosphorylation of multiple linker components.

Furthermore, Nek2 expression is positively correlated with human proliferative disease. Nek2 regulates $\beta$-catenin during centrosome separation by phosphorylation. In the cell cycle, cytoplasmic and nuclear $\beta$-catenin levels increase in the S-phase, peak at late $\mathrm{G} 2 / \mathrm{M}$, and abruptly decrease as the cells re-enter G1 after mitosis (28). Manipulating $\beta$-catenin levels, particularly in $\mathrm{G} 2$, affects centrosome maturation and function, which highlights the role of $\beta$-catenin in the centrosome (29). When cytoplasmic $\beta$-catenin is stabilized, it can translocate into the nucleus, where it interacts with a family of $\mathrm{T}$ cell factor/lymphocyte enhancer factor transcription factors to drive cell proliferation by directly inducting cell cycle regulators, such as c-Myc, CycB1, and CycD1 $(30,31)$. However, high $\beta$-catenin levels may act via the Wnt signaling pathway to stimulate cellular proliferation by dysregulating the cell cycle (high C-Myc, CycD1, and CycE expression) and inhibit tumor suppresion p16, p27, and wild-type p53; $(32,33)$.

In conclusion, Nek 2 is frequently upregulated in $\mathrm{HCC}$ compared to healthy tissues, and is associated with HCC stages. The present study demonstrates that blocking Nek 2 by siRNA inhibits HCC cell proliferation in vitro. This inhibition can be traced back to the attenuation of the expression of proteins controlling cell growth and proliferation. These results are a promising starting point for the elucidation of the functional importance of Nek2 in HCC progression. Further studies may allow Nek2 to serve as a molecular target closely associated with cell proliferation for the future development of HCC therapeutics.

\section{Acknowledgements}

The present study was supported by the National Natural Science Foundation of China (grant no. 81270037).

\section{References}

1. Meacham CE and Morrison SJ: Tumour heterogeneity and cancer cell plasticity. Nature 501: 328-337, 2013.

2. Torre LA, Bray F, Siegel RL, Ferlay J, Lortet-Tieulent J and Jemal A: Global cancer statistics, 2012. CA Cancer J Clin 65: 87-108, 2015.
3. Bodzin AS and Busuttil RW: Hepatocellular carcinoma: Advances in diagnosis, management, and long term outcome. World J Hepatol 7: 1157-1167, 2015.

4. El-Serag HB: Hepatocellular carcinoma. N Engl J Med 365: 1118-1127, 2011.

5. Slotta JE, Kollmar O, Ellenrieder V, Ghadimi BM and Homayounfar K: Hepatocellular carcinoma: Surgeon's view on latest findings and future perspectives. World J Hepatol 7: 1168-1183, 2015.

6. Oda K, Uto H, Mawatari S and Ido A: Clinical features of hepatocellular carcinoma associated with nonalcoholic fatty liver disease: A review of human studies. Clin J Gastroenterol 8: 1-9, 2015.

7. Choi KJ, Baik IH, Ye SK and Lee YH: Molecular targeted therapy for hepatocellular carcinoma: Present status and future directions. Biol Pharm Bull 38: 986-991, 2015.

8. Zhang Y: Identification of differentially expressed genes in hepatocellular carcinoma by laser capture microdissection and microarray. Guangzhou Medical University, 2009.

9. Fry AM: The Nek2 protein kinase: A novel regulator of centrosome structure. Oncogene 21: 6184-6194, 2002.

10. Schultz SJ, Fry AM, Sütterlin C, Ried T and Nigg EA: Cell cycle-dependent expression of Nek2, a novel human protein kinase related to the NIMA mitotic regulator of Aspergillus nidulans. Cell Growth Differ 5: 625-635, 1994.

11. Uto K and Sagata N: Nek2B, a novel maternal form of Nek2 kinase, is essential for the assembly or maintenance of centrosomes in early Xenopus embryos. Embo J 19: 1816-1826, 2000.

12. Fry AM, Schultz SJ, Bartek J and Nigg EA: Substrate specificity and cell cycle regulation of the Nek2 protein kinase, a potential human homolog of the mitotic regulator NIMA of Aspergillus nidulans. J Biol Chem 270: 12899-12905, 1995.

13. Fletcher L, Cerniglia GJ, Nigg EA, Yend TJ and Muschel RJ: Inhibition of centrosome separation after DNA damage: A role for Nek2. Radiat Res 162: 128-135, 2004.

14. Bowers AJ and Boylan JF: Nek8, a NIMA family kinase member, is overexpressed in primary human breast tumors. Gene 328: 135-142, 2004.

15. Kokuryo T, Senga T, Yokoyama Y, Nagino M, Nimura Y and Hamaguchi M: Nek2 as an effective target for inhibition of tumorigenic growth and peritoneal dissemination of cholangiocarcinoma. Cancer Res 67: 9637-9642, 2007.

16. Suzuki K, Kokuryo T, Senga T, Yokoyama Y, Nagino M and Hamaguchi M: Novel combination treatment for colorectal cancer using Nek2 siRNA and cisplatin. Cancer Sci 101: 1163-1169, 2010.

17. Tsunoda N, Kokuryo T, Oda K, Senga T, Yokoyama Y, Nagino M, Nimura Y and Hamaguchi M: Nek2 as a novel molecular target for the treatment of breast carcinoma. Cancer Sci 100: 111-116, 2009.

18. Drozdov I, Bornschein J, Wex T, Valeyev NV, Tsoka S and Malfertheiner P: Functional and topological properties in hepatocellular carcinoma transcriptome. Plos One 7: e35510, 2012.

19. Gu Z, Zhou W, Huang J, Yang Y, Wendlandt E, Xu H, He X, Tricot $\mathrm{G}$ and Zhan F: Nek2 is a novel regulator of B cell development and immunological response. Biomed Res Int 2014: 621082, 2014.

20. Wai DH, Schaefer KL, Schramm A, Korsching E, Van Valen F, Ozaki T, Boecker W, Schweigerer L, Dockhorn-Dworniczak B and Poremba C: Expression analysis of pediatric solid tumor cell lines using oligonucleotide microarrays. Int J Oncol 20: 441-451, 2002.

21. Andreasson U, Dictor M, Jerkeman M, Berglund M, Sundström C, Linderoth J, Rosenquist R, Borrebaeck CA and Ek S: Identification of molecular targets associated with transformed diffuse large B cell lymphoma using highly purified tumor cells. Am J Hematol 84: 803-888, 2009.

22. Marina M and Saavedra HI: Nek2 and Plk4: Prognostic markers, drivers of breast tumorigenesis and drug resistance. Front Biosci (Landmark Ed) 19: 352-365, 2014.

23. Wu W, Baxter JE, Wattam SL, Hayward DG, Fardilha M, Knebel A, Ford EM, da Cruz e Silva EF and Fry AM: Alternative splicing controls nuclear translocation of the cell cycle-regulated Nek2 kinase. J Biol Chem 282: 26431-26440, 2007.

24. Das TK, Dana D, Paroly SS, Perumal SK, Singh S, Jhun H, Pendse J, Cagan RL, Talele TT and Kumar S: Centrosomal kinase Nek2 cooperates with oncogenic pathways to promote metastasis. Oncogenesis 2: e69, 2013. 
25. Mbom BC, Nelson WJ and Barth A: $\beta$-catenin at the centrosome: Discrete pools of $\beta$-catenin communicate during mitosis and may co-ordinate centrosome functions and cell cycle progression. Bioessays 35: 804-969, 2013.

26. Rapley J, Baxter JE, Blot J, Wattam SL, Casenghi M, Meraldi P, Nigg EA and Fry AM: Coordinate regulation of the mother centriole component nlp by nek 2 and plk1 protein kinases. Mol Cell Biol 25: 1309-1324, 2005.

27. Hayward DG and Fry AM: Nek2 kinase in chromosome instability and cancer. Cancer Lett 237: 155-166, 2006.

28. Mbom BC, Siemers KA, Ostrowski MA, Nelson WJ and Barth AI: Nek2 phosphorylates and stabilizes $\beta$-catenin at mitotic centrosomes downstream of Plk1. Mol Biol Cell 25: 977-991, 2014.

29. Olmeda D, Castel S, Vilaro S and Cano A: $\beta$-catenin regulation during the cell cycle: Implications in G2/M and apoptosis. Mol Biol Cell 14: 2844-2860, 2003.
30. Gehrke I, Gandhirajan RK and Kreuzer KA: Targeting the WNT/ $\beta$-catenin/TCF/LEF1 axis in solid and haematological cancers: Multiplicity of therapeutic options. Eur J Cancer 45: 2759-2767, 2009.

31. Sekiya T, Nakamura T, Kazuki Y, Oshimura M, Kohu K, Tago K, Ohwada S and Akiyama T: Overexpression of Icat induces G(2) arrest and cell death in tumor cell mutants for adenomatous polyposis coli, $\beta$-catenin, or Axin. Cancer Res 62: 3322-3326, 2002.

32. Chou J, Lin YC, Kim J, You L, Xu Z, He B and Jablons DM: Nasopharyngeal carcinoma-review of the molecular mechanisms of tumorigenesis. Head Neck 30: 946-963, 2008.

33. Salomon A, Keramidas M, Maisin C and Thomas M: Loss of $\beta$-catenin in adrenocortical cancer cells causes growth inhibition and reversal of epithelial-to-mesenchymal transition. Oncotarget 6: 11421-11433, 2015. 